
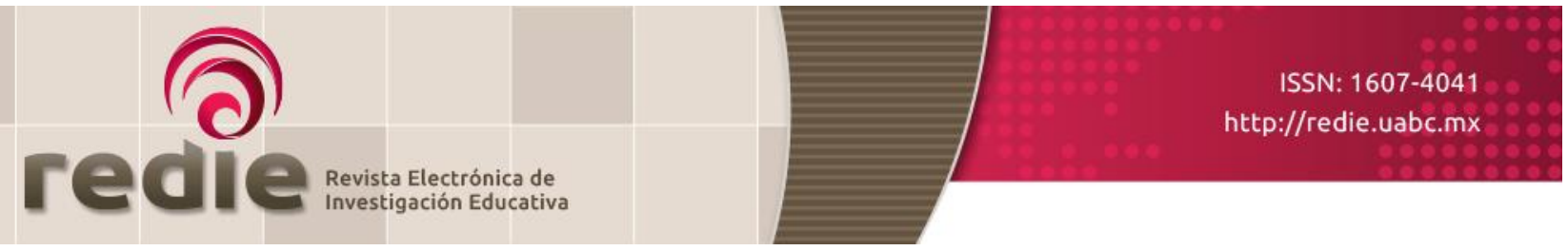

Vol. 21, 2019/e29

\title{
Un estudio sobre el rendimiento académico en Matemáticas
}

\section{A Study on Academic Achievement in Mathematics}

\author{
Jorge Daniel Mello Román (1) decanatofacet@unc.edu.py \\ Adolfo Hernández Estrada (2) adolfher@ucm.es
}

(1) Universidad Nacional de Concepción

(2) Universidad Complutense de Madrid

(Recibido: 19 de julio de 2017; Aceptado para su publicación: 5 de diciembre de 2017)

Cómo citar: Mello, J. D. y Hernández, A. (2019). Un estudio sobre el rendimiento académico en Matemáticas. Revista Electrónica de Investigación Educativa, 21, e29, 1-10. doi:10.24320/redie.2019.21.e29.2090

\section{Resumen}

El propósito de este trabajo es explicar el rendimiento académico en Matemáticas a partir de características individuales de los estudiantes. Se toma el caso particular de Paraguay, país ubicado por debajo del promedio regional en recientes evaluaciones internacionales sobre logros de aprendizaje en el área de Matemáticas por estudiantes de Educación Escolar Básica. Se aplicó un cuestionario a 899 estudiantes del tercer ciclo de Educación Escolar Básica de instituciones educativas de gestión pública y privada del distrito de Concepción. Se utilizaron dos técnicas de modelación estadística: Árbol de decisión y Regresión lineal múltiple sobre variables observables. Se concluye que el rendimiento académico en Matemáticas se explica en gran medida por el aprendizaje que logra el estudiante en el contexto del aula, así como por la percepción que tiene de su propia capacidad.

Palabras clave: Rendimiento académico, modelos estadísticos, enseñanza de Matemáticas.

\section{Abstract}

This paper seeks to explain academic achievement in Mathematics based on individual student characteristics. The study takes the case of Paraguay, a country below the regional average in recent international assessments of learning achievement in mathematics by basic-level students (students in Educación Escolar Básica). A questionnaire was administered to 899 students of the third cycle of basiclevel education $\left(7^{\text {th }}, 8^{\text {th }}\right.$, and $9^{\text {th }}$ grades) from both public and private schools in the district of Concepción. Two statistical modeling techniques were employed on observable variables: decision trees and multiple linear regression. It is concluded that academic achievement in mathematics is largely explained by students' learning in a classroom setting and perception of their own ability. 


\section{Introducción}

El rendimiento académico es un resultado del proceso de aprendizaje escolar, en el cual convergen los efectos de numerosas variables sociales, personales, y sus interrelaciones (Fullana, 2008). Actualmente, los expedientes académicos y las calificaciones de los escolares son utilizados como fuente principal para valorar los resultados de la enseñanza y constituyen el criterio para definir el rendimiento académico (Corea, 2001).

En los últimos años se han incrementado las contribuciones teóricas y trabajos empíricos con el objetivo de determinar las causas del rendimiento académico de los estudiantes. Según Planck y Aliaga (2013) hay consenso en que los factores asociados al desempeño académico pueden tener su origen en dos grandes ámbitos, los determinantes personales y los determinantes sociales. En el primero de ellos se incluyen: la inteligencia, las aptitudes, la asistencia a clases y otros. En el segundo, el entorno familiar, el contexto socioeconómico, las variables demográficas y la escolaridad de los padres, coincidiendo con Velázquez y Rodríguez (2006), quienes plantean que las variables relacionadas con el rendimiento académico pueden ser internas o externas, entendiendo las internas como las características personales del estudiante y las externas como factores del contexto.

Los informes y artículos de investigación revisados prestan una atención especial a las variables afectivas, principalmente las actitudes. El común denominador es la conclusión de que las variables afectivas son tan importantes como las variables cognitivas en su influencia en el aprendizaje, e incluso en la elección de una futura carrera universitaria y hasta en el uso del tiempo libre (Bojórquez, Quiroz y Quiroz, 2014). Varios estudios correlacionan positivamente las actitudes y el rendimiento académico en Matemáticas, así como la autoconfianza, pero intervienen aspectos como los socioculturales y otros (Ruiz y Ursini, 2010).

Según Navarro (2003), la autopercepción del alumno sobre su habilidad es el elemento central para el éxito académico y, citando a Covington (1984, p. 1), propone clasificar a los estudiantes en tres grandes grupos:

a) Los orientados al dominio. Sujetos que tienen éxito escolar, se consideran capaces, presentan alta motivación de logro y muestran confianza en sí mismos.

b) Los que evitan el fracaso. Aquellos estudiantes que carecen de un firme sentido de aptitud y autoestima y ponen poco esfuerzo en su desempeño; para "proteger" su imagen ante un posible fracaso, recurren a estrategias como la participación mínima en el salón de clases, retraso en la realización de una tarea, trampas en los exámenes, etc.

c) Los que aceptan el fracaso. Sujetos derrotistas que presentan una imagen propia deteriorada y manifiestan un sentimiento de desesperanza aprendido, es decir, que han aprendido que el control sobre el ambiente es sumamente difícil o imposible y, por lo tanto, renuncian al esfuerzo.

Fullana (2008) manifiesta que las variables que inciden sobre el rendimiento académico son tantas que, normalmente, en las investigaciones se opta por simplificar la búsqueda, enfocándolas en el estudio de las relaciones de una o dos variables con el rendimiento escolar. Aunque los resultados difieren, por lo general hay un grupo de variables que aparecen con mayor frecuencia en la mayoría de estudios, por ejemplo: género, edad, entorno sociocultural, tipo de centro educativo, hábitos de estudio, hábitos de lectura y hábitos de ocio. En todos estos casos se considera al rendimiento académico como variable dependiente de las otras variables, con lo que se establece una relación de causa-efecto.

En cuanto a los tipos de modelos de rendimiento académico, De la Orden et al. (2001) citando a López, Marqués y Martínez (1985, p. 170) plantea la siguiente clasificación:

a) Modelos centrados en el alumno: que intentan identificar la repercusión en el rendimiento escolar que tienen variables de orden somático y fisiológico y las características individuales tanto estáticas (capacidades intelectuales y de personalidad) como dinámicas (motivación, actitud, 
intereses) vinculadas más estrechamente al contexto.

b) Modelos centrados en el contexto: que incorporan factores sociales tanto de tipo estático (procedencia sociocultural y económica) como dinámico (fuerzas sociales, institucionales y culturales) que representan la red de fuerzas sociales ambientales.

c) Modelos pedagógico-didácticos: que intentan identificar las principales variables asociadas al rendimiento a través de opiniones y valoraciones de alumnos y profesores, y las características del contexto institucional próximo (clima de aula e interacciones personales) y analizar las variables metodológicas en su mutua interacción con las aptitudes de los estudiantes.

En la literatura se encuentran investigaciones sobre el rendimiento académico apoyadas en diversas técnicas de modelación estadística. Por ejemplo, Porcel, Dapozo y López (2010) formularon un modelo de regresión logística binaria a fin de predecir el rendimiento académico de alumnos de primer año en una universidad argentina a partir de características socioeducativas de los estudiantes, mientras que Hernández y González (2011) proponen un modelo de ecuaciones estructurales que evalúa los efectos diferenciados que los factores económicos, sociales y culturales tienen sobre el logro académico.

El presente estudio toma el caso particular de Paraguay, país en el que se realizaron varias mediciones del desempeño escolar desde inicios de este siglo, tanto nacionales como internacionales, siendo los resultados en el área de Matemáticas, en todos los casos, bajos.

El Segundo Estudio Regional Comparativo y Explicativo (SERCE), realizado entre 2004 y 2008, fue una de las primeras mediciones internacionales en las que participó el país. En esta medición aplicada en 3o. y 60. grados, el Paraguay fue uno de los cinco países que obtuvo puntajes por debajo del promedio en todas las materias evaluadas: Lenguaje, Matemáticas y Ciencias. Los resultados obtenidos por el país en el Tercer Estudio Regional Comparativo y Explicativo (TERCE), desarrollado entre 2010 y 2014, evidencian un retroceso en la calidad del aprendizaje en la Educación Primaria, representado en un -3\% en el promedio global obtenido. En el área Matemáticas, para 3o. se obtuvo un promedio similar al SERCE y para el 6o. un promedio significativamente más bajo (UNESCO, 2014).

En Paraguay, el tercer ciclo de la Educación Escolar Básica (EEB) (70., 80. y 9o. grados) integra al sistema educativo nacional a pre-adolescentes y adolescentes de entre 12 y 15 años, aproximadamente. El diseño curricular nacional (Ministerio de Educación y Cultura [MEC], 2010) establece como competencia del área de Matemáticas para este ciclo:

Formula y resuelve situaciones problemáticas que involucren la utilización de: operaciones matemáticas con números reales y expresiones algebraicas; unidades de medidas; conceptos, principios y elementos de la Geometría plana y del espacio; procedimientos básicos de la Estadística descriptiva y de la Probabilidad, en variados contextos (p. 54).

El objetivo de esta investigación es estudiar las características individuales de los estudiantes que mejor explican el rendimiento académico en Matemáticas en el tercer ciclo de la EEB, para ello se emplearon dos técnicas de modelación estadística: Árbol de decisión y Regresión lineal múltiple.

\section{Método}

La población objetivo estuvo constituida por estudiantes de entre 12 y 15 años inscritos en el tercer ciclo de la EEB de instituciones educativas de gestión pública y privada del distrito de Concepción, República del Paraguay. El marco muestral estuvo conformado por 30 instituciones educativas con 2,542 estudiantes matriculados en el 2016, conforme a registros oficiales proveídos por la Coordinación Departamental de Supervisiones Educativas de Concepción, dependencia regional del MEC del país.

Se utilizó el muestreo probabilístico por conglomerados de una etapa. Fueron aplicados los procedimientos señalados en Bencardino (2012), considerando como conglomerados a las instituciones 
educativas. En total fueron seleccionadas 16 instituciones y encuestados 899 estudiantes durante los meses de agosto y setiembre de 2016. La muestra se resume en la tabla I.

Tabla I. Instituciones educativas seleccionadas y cantidad de estudiantes encuestados

\begin{tabular}{l|c}
\hline Instituciones educativas & $\begin{array}{c}\text { Cantidad de } \\
\text { estudiantes }\end{array}$ \\
\hline Esc. Bas. Subv. Santa Teresita & 57 \\
\hline Esc. Bas. Subv. Campo Verde & 59 \\
\hline Esc. Bas. Subv. Centro Educacional Presb. & 27 \\
Esc. Bas. Virgen Del Rosario & 41 \\
\hline Esc. Bas. Gral. José E. Díaz & 104 \\
\hline Esc. Bas. San Isidro Labrador & 44 \\
\hline Esc. Bas. Prof. Jorge S Miranda & 79 \\
Colegio Nacional AgustínFern. Pinedo & 10 \\
\hline Esc. Bas. Agustín Fernando De Pinedo & 103 \\
\hline Esc. Bas. Subv. Inmaculada Concepción & 73 \\
\hline Liceo. Nac. Mons. MaricevichFleitas & 82 \\
Colegio Departamental Concepción & 49 \\
\hline Esc. Bas. Priv. Subv. San Francisco & 76 \\
Esc. Bas. Mayor E. J. Planas & 47 \\
\hline Esc. Bas. José de Antequera y Castro & 32 \\
\hline Esc. Bas. Priv. Subv. Juan Pablo ll & 16 \\
\hline Total & 899 \\
\hline
\end{tabular}

El instrumento de medición aplicado fue el cuestionario que se describe con detalle en la tabla II, que permitió recoger de manera organizada los indicadores de las variables implicadas (Anguita, Labrador y Campos, 2003). Este cuestionario ha sido elaborado con base en las encuestas para alumnos del programa CRECER del Ministerio de Educación de Perú implementado en 1998 (Ministerio de Educación del Perú, 2017) y ajustado al contexto educativo y sociocultural del Paraguay, y contiene preguntas que miden 10 variables: Rendimiento Académico, Sexo, Edad, Aprendizaje en Aula, Bilingüismo (CastellanoGuaraní), Aprendizaje Autónomo, Autoconcepto Académico, Tutorías Externas, Expectativa de Futuro y Condición Laboral.

Se optó por una cantidad reducida de variables a fin de garantizar la parsimonia de los modelos a construir. La tabla II recoge la definición operacional de las variables involucradas y los indicadores o preguntas utilizadas para la medición. 
Tabla II. Cuadro operacional de variables

\begin{tabular}{|c|c|c|c|}
\hline Variables & Definición Operacional & Preguntas formuladas & $\begin{array}{c}\text { Medida: } \\
\text { Respuestas posibles }\end{array}$ \\
\hline $\begin{array}{l}\text { Rendimiento } \\
\text { académico }\end{array}$ & $\begin{array}{l}\text { Calificación numérica obten } \\
\text { en evaluación sumativa. }\end{array}$ & $\begin{array}{l}\text { ¿Qué calificación obtuviste en } \\
\text { Matemáticas en la última evaluación } \\
\text { global que tuviste? }\end{array}$ & Escala: 1 al 5 \\
\hline Sexo & $\begin{array}{l}\text { Característica fisiológica que } \\
\text { distingue hombres y mujeres. }\end{array}$ & ¿De qué sexo eres? & Nominal: Hombre, Mujer \\
\hline Edad & Cantidad de años cumplidos. & ¿Cuántos años tienes? & $\begin{array}{l}\text { Escala: (Rango no } \\
\text { definido) }\end{array}$ \\
\hline $\begin{array}{l}\text { Aprendizaje } \\
\text { en aula }\end{array}$ & $\begin{array}{l}\text { Percepción sobre su aprendizaje } \\
\text { durante el desarrollo de las } \\
\text { clases. }\end{array}$ & $\begin{array}{l}\text { ¿Cuánto aprendes durante el } \\
\text { desarrollo de las clases de } \\
\text { Matemáticas? }\end{array}$ & $\begin{array}{l}\text { Ordinal: Nada, Algunas } \\
\text { Cosas, Casi Todo, Todo }\end{array}$ \\
\hline $\begin{array}{l}\text { Bilingüismo } \\
\text { (Castellano- } \\
\text { Guaraní) }\end{array}$ & $\begin{array}{l}\text { Coincidencia de lenguaje entre } \\
\text { entorno escolar y familiar. }\end{array}$ & $\begin{array}{l}\text { ¿Qué idioma se habla más en tu } \\
\text { casa? ¿En qué idioma te habla tu } \\
\text { profesor de Matemáticas? }\end{array}$ & $\begin{array}{l}\text { Nominal: Castellano, } \\
\text { Guaraní, Ambos. }\end{array}$ \\
\hline $\begin{array}{l}\text { Aprendizaje } \\
\text { autónomo }\end{array}$ & $\begin{array}{l}\text { Regularidad en la realización de } \\
\text { tareas escolares. }\end{array}$ & $\begin{array}{l}\text { En una semana ¿cuántos días haces } \\
\text { tareas de Matemáticas en tu casa? }\end{array}$ & $\begin{array}{l}\text { Escala: (Rango no } \\
\text { definido) }\end{array}$ \\
\hline $\begin{array}{l}\text { Autoconcepto } \\
\text { académico }\end{array}$ & $\begin{array}{l}\text { Percepción sobre su capacidad } \\
\text { cognitiva en relación con la } \\
\text { asignatura. }\end{array}$ & $\begin{array}{l}\text { ¿Consideras que te cuesta aprender } \\
\text { Matemáticas? }\end{array}$ & $\begin{array}{l}\text { Nominal: Si (Negativo), } \\
\text { No (Positivo) }\end{array}$ \\
\hline $\begin{array}{l}\text { Tutorías } \\
\text { externas }\end{array}$ & $\begin{array}{l}\text { Frecuencia de tutorías } \\
\text { personalizadas externas a la } \\
\text { institución. }\end{array}$ & $\begin{array}{l}\text { ¿Recurres a profesores particulares } \\
\text { раra aprender mejor Matemáticas? }\end{array}$ & $\begin{array}{l}\text { Ordinal: Nunca, A veces, } \\
\text { A menudo, Siempre }\end{array}$ \\
\hline $\begin{array}{l}\text { Expectativa } \\
\text { de futuro }\end{array}$ & $\begin{array}{l}\text { Expectativa hacia su futuro, } \\
\text { asociada a factores socio- } \\
\text { ambientales y culturales. }\end{array}$ & $\begin{array}{l}\text { ¿Qué te gustaría hacer al concluir el } \\
\text { bachillerato? }\end{array}$ & $\begin{array}{l}\text { Nominal: Seguir una } \\
\text { carrera universitaria, } \\
\text { Trabajar, Tener un oficio, } \\
\text { No sabe, Otros. }\end{array}$ \\
\hline $\begin{array}{l}\text { Condición } \\
\text { laboral }\end{array}$ & Situación de trabajo infantil & $\begin{array}{l}\text { ¿Trabajas para contribuir con los } \\
\text { gastos de la casa? }\end{array}$ & Nominal: Si, No \\
\hline
\end{tabular}

En Paraguay, sus habitantes se comunican a través de dos idiomas predominantes o una combinación de ambos: el Castellano y el Guaraní (Blestel, 2015). Se agrega la variable Bilingüismo a fin de determinar si la variación del lenguaje en los entornos familiar y escolar incide en el Rendimiento académico en Matemáticas.

La encuesta fue administrada por estudiantes de la carrera de Matemáticas Aplicadas de la Universidad Nacional de Concepción, quienes actuaron como facilitadores. En la etapa de preprocesamiento de datos fueron excluidos 78 casos por presentar registros incompletos con relación a una o más variables, por lo que el conjunto de datos final está integrado por 821 casos. Los datos fueron procesados con ayuda del paquete informático SPSS.

\subsection{Técnicas de Modelación Estadística}

Para este estudio se utilizaron dos técnicas de modelación estadística:

Árbol de decisión, técnica estadística que genera un modelo de clasificación basado en diagramas de flujo, para identificar grupos, descubrir las relaciones entre grupos y realizar inferencias. Según BerlangaSilvente, Rubio-Hurtado y Baños (2013), un Árbol de decisión ayuda a tomar la decisión más "acertada" desde un punto de vista probabilístico ante un abanico de posibles decisiones, permite examinar los resultados y determinar visualmente cómo fluye el modelo, e inclusive hallar relaciones y subgrupos más específicos.

Se ha escogido el método de división Chi cuadrado $\left(x^{2}\right)$, algoritmo de árbol estadístico y multidireccional que explora datos de forma rápida y eficaz, y crea segmentos y perfiles con respecto al resultado deseado. Permite la detección automática de interacciones mediante Chi cuadrado y se elige en cada paso la variable independiente (predictor) que presenta la interacción más fuerte con la variable 
dependiente (Berlanga-Silvente et al., 2013).

Regresión lineal múltiple, técnica estadística que se utiliza con propósitos de explicación y predicción: el primero para identificar las variables independientes que influyen en la o las variables dependientes; el segundo, para conocer qué valores de la variable respuesta son más probables, dado un conjunto de valores de las variables independientes (Carreto, Jaimes, Hernández, Rosas e Ignacio, 2014).

En cuanto al uso de la Regresión lineal múltiple en estudios sobre el rendimiento académico, De la Orden, Olivero, Mafokozi y González (2001) señalan que la regresión lineal trata de establecer la contribución independiente de diversas variables sobre la variable criterio, representada por las calificaciones escolares, los resultados obtenidos en pruebas objetivas de rendimiento, etc. La variación total de la variable Rendimiento puede dividirse en tres partes: la varianza que se atribuye a cada variable explicativa de forma independiente, la varianza que se atribuye al conjunto de todas las variables predictivas, y la parte de varianza que no puede explicarse por el modelo de regresión o varianza residual.

\section{Resultados}

A continuación, se describen los resultados de las técnicas de modelación estadística descritas, tomando en ambos casos como variable dependiente el Rendimiento académico y las restantes 9 variables como independientes.

\subsection{Modelación Estadística por Árbol de decisión}

El Árbol de decisión generado con el paquete informático sPSS sobre el conjunto de datos descrito en el método se presenta en la figura 1. En ella se aprecia que el mejor predictor del rendimiento académico en Matemáticas es el nivel de aprendizaje en el entorno escolar indicado por la variable Aprendizaje en Aula. La media del rendimiento académico se sitúa en 4,076 para los estudiantes que manifiestan entender "Todo" durante el desarrollo de las clases de Matemáticas, en contraste a una media de 2,411 para aquellos que manifiestan aprender "Solo algunas cosas" o "Nada".

La variable Autoconcepto académico evidencia una influencia positiva en los niveles de Rendimiento académico cuando la variable Aprendizaje en aula no se encuentra en el valor superior, es decir, a pesar que el estudiante no comprenda "todo" en clase, una percepción positiva de su propia capacidad de aprendizaje en Matemáticas eleva la media del Rendimiento académico. 


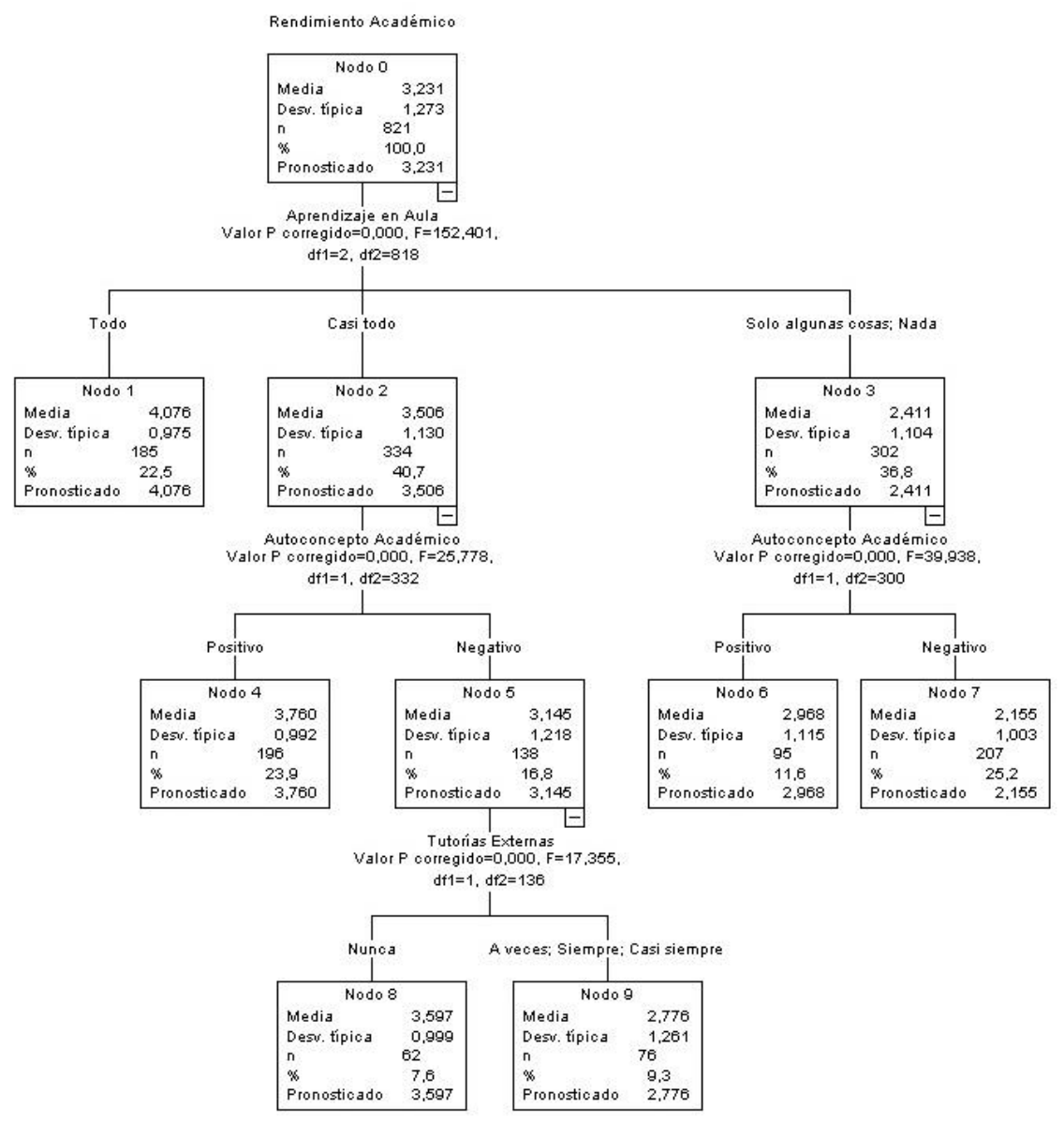

Figura 1. Árbol de decisión. Variable dependiente: Rendimiento académico

Un aspecto relevante es la relación negativa de la variable Tutoría externa, con el nivel de Rendimiento académico y el Autoconcepto académico, pues los estudiantes que recurren con cierta frecuencia a tutoría de personas externas a las instituciones educativas para aprender mejor Matemáticas, denominados comúnmente en el país "profesores particulares", no registran elevados niveles de rendimiento académico y además tienen una percepción negativa de su propia capacidad de aprendizaje.

La técnica empleada no valora como significativa la relación de las variables: Sexo, Edad, Bilingüismo, Aprendizaje autónomo, Expectativa de futuro y Condición laboral, con el Rendimiento académico.

El modelo estadístico así construido permite diferenciar claramente tres grupos de estudiantes: 1) Estudiantes orientados al dominio, con elevados niveles de rendimiento académico y que se consideran capaces, 2) Estudiantes que evitan el fracaso, sin un firme sentido de autoestima y que recurren a estrategias que no contemplan necesariamente un mayor esfuerzo personal y 3) Estudiantes que aceptan el fracaso, con bajos niveles de rendimiento académico y una imagen propia deteriorada. Este resultado constituye una aproximación empírica a la clasificación teórica propuesta por Covington (1984).

\subsection{Modelación Estadística por Regresión lineal múltiple}

El modelo de Regresión lineal múltiple fue generado con ayuda del paquete informático spss sobre el mismo conjunto de datos. Se ha escogido como método de selección de variables a incluir el de "Pasos hacia adelante". 
El valor del Coeficiente de Determinación obtenido fue $\mathrm{R}^{2}=37.9 \%$, que representa la proporción de variabilidad del Rendimiento académico que se atribuye al conjunto de todas las variables predictoras incluidas en el modelo. Considerando la escala del 1 al 5 en el Rendimiento académico, el $\mathrm{R}^{2}$ obtenido representa una variabilidad de hasta 2 puntos $(5 \times 37.9 \%=1,895 \approx 2)$ explicada por el modelo generado.

El resumen del modelo generado se presenta en la tabla III. Las columnas: Suma de Cuadrados, Grados de libertad ( $g($ ) y Cuadrado Medio, son las habituales en todo Análisis de Varianza; el estadístico F de FisherSnedecor permite extraer las variables estadísticamente significativas para el modelo generado, aquellas cuyo correspondiente $p$-valor es inferior a un nivel de significación $a=0.05$.

La importancia de cada predictor representa la porción del coeficiente de determinación $\left(R^{2}\right)$ que se debe a la inclusión del mismo, y se calcula considerando la reducción de la suma de cuadrados de los residuos que resulta de incluir cada predictor y la posterior normalización de los valores obtenidos. Los procedimientos de cálculo del coeficiente se detallan en Meyers, Gamst y Guarino (2013).

Tabla III. Resumen del Modelo de Regresión lineal múltiple

\begin{tabular}{l|c|c|c|c|c|c}
\hline \multicolumn{1}{c|}{ Origen } & $\begin{array}{c}\text { Suma de } \\
\text { Cuadrados }\end{array}$ & $g l$ & $\begin{array}{c}\text { Cuadrado } \\
\text { Medio }\end{array}$ & $\mathrm{F}$ & $\rho$-valor & $\begin{array}{c}\text { Importa } \\
\text { ncia }\end{array}$ \\
\hline Modelo corregido & 511,999 & 8 & 63,950 & 63,603 & 0.000 & \\
Aprendizaje en aula & 159,641 & 2 & 79,820 & 79,387 & 0.000 & 0.521 \\
Autoconcepto académico & 70,660 & 1 & 70,660 & 70,276 & 0.000 & 0.231 \\
\hline Tutoría externas & 41,225 & 1 & 41,225 & 41,001 & 0.000 & 0.135 \\
Expectativa de futuro & 21,585 & 2 & 10,793 & 10,734 & 0.000 & 0.070 \\
Aprendizaje autónomo & 9,234 & 1 & 9,234 & 9,184 & 0.003 & 0.030 \\
Condición laboral & 3,967 & 1 & 3,967 & 3,945 & 0.047 & 0.013 \\
\hline Residuo & 816,812 & 812 & 1,005 & & & \\
\hline Total corregido & $1,328,029$ & 820 & & & & \\
\hline
\end{tabular}

Se destacan como variables de mayor importancia para predecir el Rendimiento académico: Aprendizaje en aula, Autoconcepto académico y Tutorías externas. De menor relevancia, pero igualmente incluidas en el Modelo de Regresión lineal múltiple son las variables: Expectativa de Futuro, Aprendizaje Autónomo y Condición Laboral. Con esta técnica se vuelven a valorar como poco importantes para predecir el rendimiento académico las variables: Sexo, Edad y Bilingüismo. Según las evidencias estadísticas, la coincidencia o no de la lengua dominante en el hogar y en la clase de Matemáticas, no incide significativamente en el rendimiento académico en Matemáticas, así como el Sexo y la Edad de los estudiantes del tercer ciclo de la EEB.

El modelo lineal generado permite identificar las principales variables asociadas al rendimiento académico en Matemáticas a través de opiniones de los propios alumnos, la percepción de su propio aprendizaje en el aula y sus características individuales dinámicas, motivación y actitudes vinculadas al contexto. En la clasificación de López et al. (1985) se ajusta a un modelo mixto, pedagógico-didáctico y centrado en el alumno.

\section{Discusión}

Los modelos estadísticos por Árbol de decisión y Regresión lineal múltiple coinciden en que las variables que explican el nivel de Rendimiento académico en Matemáticas son el Aprendizaje en aula y el Autoconcepto académico. La calificación escolar se relaciona con la valoración que tiene el estudiante sobre: el nivel de aprendizaje que logra en la sala de clases y el grado de dificultad que representa aprender la asignatura.

Se identifican tres grupos de estudiantes: aquellos que confían en sus propias capacidades y demuestran habilidades, aquellos que no tienen un firme sentido de autoestima, con esfuerzo mínimo en el aprendizaje y que esperan que las soluciones pasen por otros medios, y por último aquellos que subestiman sus capacidades y aceptan el bajo rendimiento sin interponer esfuerzo alguno. Resulta claro 
que es en el contexto escolar donde debe lograrse el aprendizaje y la confianza en la capacidad de aprender.

Se recomienda evaluar el diseño curricular nacional para el área Matemáticas desde su capacidad de intervenir en diferentes facetas, desde las competencias cognitivas hasta las actitudes, el bienestar e inclinación hacia el aprendizaje de los estudiantes. Futuras investigaciones podrían evaluar el efecto sobre el rendimiento académico, de factores sociales, económicos y culturales del contexto paraguayo.

\section{Referencias}

Anguita, J. C., Labrador, J. R. y Campos, J. D. (2003). La encuesta como técnica de investigación. Elaboración de cuestionarios y tratamiento estadístico de los datos (I). Atención primaria, 31(8), 527-538.

Bencardino, C. M. (2012). Estadística y muestreo. Colombia: Ecoe Ediciones.

Berlanga-Silvente, V., Rubio-Hurtado, M. J. y Baños, R. V. (2013). Cómo apolicar árboles de decisión en SPSS. Revista d'Innovació i Recerca en Educació, 6(1), 65-79. Recuperado de http://revistes.ub.edu/index.php/REIRE/article/view/reire2013.6.1615

Blestel, É. (2015). Guaraní aquí. Jopara allá: Reflexiones sobre la (socio) lingüística paraguaya (Fondo hispánico de lingüística y filología, 19). Berna, Suiza: Peter Lang.

Bojórquez, L. R., Quiroz, A. U. y Quiroz, V. M. U. (2014, julio). Las actitudes positivas y negativas de los estudiantes en el aprendizaje de las matemáticas, su impacto en la reprobación y la eficiencia terminal. 2do. Congreso Internacional: Espacio Común de Formación Docente.

Carreto, R. R., Jaimes, F. G., Hernández, F. J. A., Rosas, F. S. y Ignacio, O. F. T. (2014). Un modelo empírico para explicar el desempeño académico de estudiantes de bachillerato. Perfiles Educativos, 36(146), 45-62. Recuperado de https://www.iisue.unam.mx/perfiles/numeros/2014/146

Corea, N. C. (2001). Régimen de vida de los escolares y rendimiento académico. Tesis doctoral. Universidad Autónoma de Barcelona. Recuperado de http://www.tdx.cat/handle/10803/5002

Covington, M. (1984). The motive for self-worth. En R. Ames y C. Ames (Eds.), Research on Motivation in Education. Student Motivation. Nueva York: Academic Press.

De la Orden, A., Olivero, L., Mafokozi, J. y González, C. (2001). Modelos de investigación del bajo rendimiento. Revista Complutense de Educación, 12(1), 159-178. Recuperado de https://revistas.ucm.es/index.php/RCED/article/view/RCED0101120159A/16866

Fullana, J. (2008). La investigació sobre l'exit i el fracàs escolar des la perspectiva dels factors de risc. Implicacions per a la recerca i la pràctica educatives [La investigación sobre el éxito y el fracaso escolar desde la perspectiva del factors de riesgo. Implicaciones para la investigación y la práctica educativa]. Tesis Doctoral, Universidad de Girona. Recuperado de https://www.tesisenred.net/handle/10803/7980

Hernández, E. y González, M. J. (2011). Modelo de ecuación estructural que evalúa las relaciones entre el estatus cultural y económico del estudiante y el logro educativo. Revista Electrónica de Investigación Educativa, 13(2), 188-203. Recuperado de https://redie.uabc.mx/redie/article/view/290

López, A., Marqués, J. y Martínez, A. (1985). El fracaso escolar-percepciones y vivencias. Valencia, España: Institució Alfons El Magnánim.

Meyers, L. S., Gamst, G. C. y Guarino, A. J. (2013). Performing data analysis using IBM SPSS. Australia: John Wiley \& Sons. 
Ministerio de Educación del Perú (2017). CRECER 98, Medición de la Calidad de los Aprendizajes. Recuperado de http://umc.minedu.gob.pe/crecer-98/

Ministerio de Educación y Cultura. (2010). Programas de Estudios. Área de Matemáticas. 7o., 80., 9o. grados. Asunción, Paraguay: Autor.

Navarro, R. E. (2003). El rendimiento académico: concepto, investigación y desarrollo. Revista Electrónica Iberoamericana sobre Calidad, Eficacia y Cambio en Educación, 1(2), 1-15. Recuperado de http://www.redredial.net/referencia-bibliografica-36504.html

Planck, B. y Aliaga, V. (2013). Variables predictoras del rendimiento académico de los alumnos de primer año de las carreras de Humanidades de la Universidad de Atacama, Chile. Revista Internacional de Investigación en Ciencias Sociales, 9(2), 207-220. Recuperado de http://revistacientifica.uaa.edu.py/index.php/riics/article/view/178

Porcel, E. A., Dapozo, G. N. y López, M. V. (2010). Predicción del rendimiento académico de alumnos de primer año de la FACENA (UNNE) en función de su caracterización socioeducativa. Revista Electrónica De Investigación Educativa, 12(2), 1-21. Recuperado de https://redie.uabc.mx/redie/article/view/264

Ruiz, J. S. y Ursini, S. (2010). Actitudes hacia las matemáticas y matemáticas con tecnología: estudios de género con estudiantes de secundaria. Revista Latinoamericana de Investigación en Matemática Educativa, 13(4-II), 303-318.

UNESCO. (2014). Primera entrega de resultados TERCE: comparación de resultados del segundo y tercer estudio regional comparativo y explicativo SERCE y TERCE 2006-2013. Santiago de Chile: Autor. 